\title{
EL RIESGO DE LA POBLACIÓN A INUNDACIONES POR LLUVIAS COMO CONSECUENCIA DE LA DINÁMICA DE EXPANSIÓN URBANA SOBRE PAISAJES ANEGADIZOS. EL CASO DE LA CIUDAD DE CORRIENTES (ARGENTINA)
}

\author{
The risk of flooding rains population as a result of the dynamics of urban \\ expansion on flood landscapes. The case of Corrientes city (Argentina)
}

\section{Félix I. Contreras* y María A. Fantín**}

\section{Resumen}

El crecimiento espacial de la ciudad de Corrientes, Argentina, se ve limitado, en primer lugar, por el río Paraná que imposibilita el crecimiento de la ciudad hacia el norte y el oeste; en tanto hacia el este y el sur, predominan paisajes anegadizos.

El objetivo de este trabajo es estudiar la exposición de la población frente a riesgos por inundación urbana, como consecuencia de la expansión de la ciudad de Corrientes sobre espacios anegables. La metodología empleada consistió en la observación y análisis de imágenes satelitales que permiten analizar cambios recientes, como así también la observación de fotografías aéreas tomadas en el año 1950 y una revisión histórica de la ocupación de nuevos espacios mediante la observación de planos antiguos.

$<$ Crecimiento espacial $<$ Dinámica de expansión $><$ Ciudad y paisaje $><$ Corrientes $><$ Argentina $>$

\section{Abstract}

The spatial growth of Corrientes city, Argentina, is limited, firstly, by the Parana River that prevents its expansion toward the north and the west; whereas to the east and to the south, waterlogged landscapes predominate.

The aim of this paper is to study the exposure of the population faced with risks to urban flooding as a result of the expansion of the city of Corrientes on flooded spaces. The methodology employed consisted of the observation and analysis of satellite images that allow the detection of recent changes, as well as the observation of aerial photographs obtained in 1950, and a historical review of the occupation of new spaces by the observation of old planes.

$<$ Spatial growth dynamic expansion $><$ city and landscape $><$ Corrientes $><$ Argentina $>$

Recibido: 18/02/2015 // Aprobado: 27/04/2015

\footnotetext{
* Profesor en Geografía, Becario Doctoral (CONICET - UNNE), Centro de Ecología Aplicada del Litoral (CONICET - UNNE), figcontreras@hotmail.com

${ }^{* *}$ Doctora en Demografía, Profesora en Geografía, Investigadora Independiente, Instituto de Investigaciones Geohistóricas (UNNE-CONICET), mafantin@gmail.com
} 
Contreras y Fantin. El riesgo de la población a inundaciones por lluvias como consecuencia de la dinámica...

\section{Introducción}

Las ciudades de la región Norte Grande Argentino (conformada por las provincias de Misiones, Corrientes, Chaco, Formosa, Santiago del Estero, Tucumán, Jujuy, Salta y Catamarca (Figura 1)), experimentaron un fuerte crecimiento a partir de la segunda mitad del siglo XX, fundamentalmente por el aporte de población procedente de la migración rural y de núcleos urbanos menores ante los sucesivos períodos de crisis de los diferentes cultivos y los cambios operados en la estructura productiva de las economías provinciales (Mignone, 2010). Por otra parte, se han registrados procesos de relocalización espacial de la misma población urbana, a veces en forma planificada y en otras oportunidades de manera informal, lo que ha llevado a la expansión de las áreas urbanizadas (Meichtry y Mignone, 2003).

El crecimiento sobre sectores periféricos o sin urbanizar genera especulación inmobiliaria por parte de empresas, agencias gubernamentales, intereses privados, etc., así como también por parte de los nuevos moradores localizados en dichos terrenos. En particular, a partir del año 2000 se produjo un aumento importante del número de instalaciones informales en terrenos privados, "generando verdaderos conflictos entre los propietarios legales, los nuevos ocupantes y los organismos estatales" (Mignone, 2008).

Asimismo, es evidente que las ciudades no suelen crecer en sectores concéntricos sucesivos. "Lo hacen, en general, a lo largo de ejes de transporte, a partir de núcleos pequeños disgregados sobre el territorio que se van extendiendo. Este proceso supone una enorme fragmentación de los sistemas naturales o seminaturales y deterioro de su potencial para prestar servicios ecológicos y mantener la biodiversidad" (Terradas et. al., 2011).

En otras ocasiones, la expansión urbana mantiene una cierta dirección debido a la presencia de barreras naturales, como un río, o propias de las actividades de la población, tales como un aeropuerto o una vía ferroviaria. En estos casos, se produce una demanda desmedida de los mejores espacios disponibles, generando marcados contrastes en la ocupación del espacio y la valorización del suelo. La expansión urbana más precaria es la que suele quedar expuesta a determinados riesgos naturales, situación en la cual, según Chardon (1998) “intervienen dos componentes principales: el fenómeno natural potencialmente dañino (la amenaza) y la sociedad amenazada susceptible de sufrir los daños (la sociedad vulnerable)". Sin embargo, estas situaciones de exposición al riesgo en un medio urbano pueden ser prevenidas si se realiza un ordenamiento territorial local.

Según Berrenechea et al (2000), habitualmente sólo se pone el énfasis en los aspectos físicos naturales desencadenantes y en la magnitud del daño producido en cada caso, lo que permite dar cuenta de otras dimensiones que hacen a la complejidad del problema en cuestión, cuya consideración permitiría logar una disminución de las consecuencias catastróficas. En ese sentido (Natenzon, 1995: 2) ha identificado cuatro dimensiones íntimamente relacionadas entre sí:

- La peligrosidad, que se refiere al potencial peligroso que tiene los fenómenos naturales (espontáneos o manipulados). Una forma de manejar la peligrosidad 
es conocerla, para lo cual el aporte de las ciencias naturales básicas y aplicadas es imprescindible.

- La vulnerabilidad, que se encuentra definida por las condiciones socioeconómicas previas a la ocurrencia del evento catastrófico.

- La exposición, que se refiere a la distribución de lo que es "potencialmente" afectable, la población y los bienes materiales "expuestos" al fenómeno peligroso. Es una consecuencia de la interrelación entre peligrosidad y vulnerabilidad, y -a la vez- incide sobre ambas. Esta componente se expresa territorialmente como construcción histórica que entrelaza los procesos físicos naturales con las relaciones socioeconómicas, configurando determinados usos de suelo y distribución de la infraestructura, asentamientos humanos, servicios públicos, etc.

- La incertidumbre, que se relaciona con las limitaciones en el estado del conocimiento (incertidumbre técnica) y las indeterminaciones en cuanto a competencias institucionales y aspectos normativos (incertidumbre social); al mismo tiempo, estas limitaciones y la complejidad de los fenómenos en cuestión impiden el manejo de la totalidad de variables involucradas, impregnando de incertidumbre los procesos de toma de decisiones.

A su vez destacan que con frecuencia los autores referidos a riesgos y desastres asocien a la vulnerabilidad íntimamente ligada con el concepto de pobreza que, como señalan Blaikie et al (1998), la vulnerabilidad y la pobreza no son sinónimos.

Olcina Cantos (2004) menciona que en España existen dos posturas principales en el análisis del peligro de inundaciones durante los últimos años, posturas que se manifiestan en Corrientes y en otras provincias de Argentina.

"Por un lado, la postura "técnica-administrativa" que defiende que se estarían produciendo más episodios de inundación por causa climática- que son fruto de la "impresibilidad", esto es, del azar de la propia naturaleza y ello traería como consecuencia la génesis de mayores desastres. Por otra, la postura "ético-geográfica" según la cual, en virtud del análisis de la realidad territorial de cada espacio geográfico a partir del trabajo de campo, el riesgo ante episodios de inundación se habría incrementado, sobremanera, en los último lustros merced a la implantación de los usos urbanos y de ocio poco acordes con los rasgos físicos del medio y ello habría provocado un aumento de la vulnerabilidad de las poblaciones asentadas en territorios de riesgo".

Para Berrenechea et al (2000) es importante señalar que si tomamos sólo el momento de la catástrofe (corte sincrónico), el fenómeno puede afectar a distintos 
Contreras y Fantin. El riesgo de la población a inundaciones por lluvias como consecuencia de la dinámica...

grupos sociales sin importar su condición socioeconómica (por ejemplo, una explosión o una inundación no prevista). En cambio, si se hace un análisis diacrónico, que considera a las catástrofes como actualización del riesgo, en el antes, en el durante y en el después del desastre, las condiciones concretas en las que cada grupo se puede preparar o reconstruir son muy diferentes.

Asumir el carácter reflexivo de los actores de la modernidad desplaza el centro de la atención de la fatalidad hacia la responsabilidad propia. Así, se modifica la percepción del problema y los riesgos pasan de ser considerados una situación fatal externa a ser asumidos como responsabilidad de los actores, sus actos y decisiones. En consecuencia, los desastres no pueden considerarse obras divinas, fortuna o fatalidad (Giddens, 1990).

Según Clichvsky (2006: 40), en la actualidad "las inundaciones en ciudades argentinas se producen por lluvia y desbordes, debido a la existencia de actividades urbanas en zonas que siempre fueron inundables y a las modificaciones del comportamiento del sistema hídrico por efecto de la ocupación del suelo. Desde 1985 hasta marzo de 1998 se produjeron, sólo en la ciudad de Buenos Aires, un total de 26 procesos de inundación por precipitación y desborde del sistema de desagües".

En función de lo expuesto, el objetivo de este trabajo es estudiar la exposición de la población frente a riesgos por inundación urbana, como consecuencia de la expansión de la ciudad de Corrientes sobre espacios anegables.

\section{Área de estudio}

La ciudad de Corrientes es la población más antigua del nordeste argentino. Su fundación fue realizada por Juan Torres de Vera y Aragón el 3 de abril de 1588, como parte de las obligaciones contraídas por el adelantado de la corona española, Juan Ortiz de Zárate. Su ubicación era estratégica y aseguraba un puerto a las comunicaciones fluviales entre Asunción y el Río de la Plata. Ya en 1581, Fray Juan de Rivadeneyra, había advertido su privilegiada ubicación geográfica (Maeder y Gutiérrez, 2003).

El sitio del emplazamiento presenta inconvenientes, dado que está limitado por el río Paraná al norte y al oeste, lo que obliga que el crecimiento se dirija hacia el E, SE y S. No obstante, éste no es el único condicionante, ya que la ciudad está rodeada de bañados y esteros, motivo por el cual su crecimiento espacial se orientó preferentemente hacia la Lomada Norte, que se distingue del terreno circundante por presentar mayores alturas, suelos con buen drenaje y un paisaje de pastizales con isletas boscosas (Figura 1). Esta lomada presenta, además, numerosas lagunas, algunas de las cuales pueden evaporar por completo el agua de sus cubetas en períodos secos dejando espacios que son ocupados por la población, usualmente en forma espontánea o a causa de una escasa, nula o incorrecta planificación inmobiliaria. En los períodos lluviosos pueden presentarse, por lo tanto, distintas problemáticas. 
Figura 1.Fotografía del paisaje de la Lomada Norte

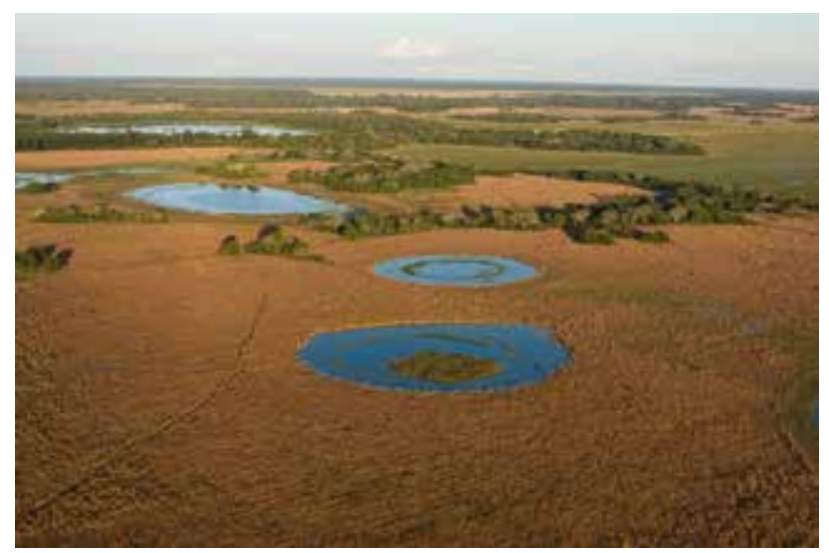

Fuente: Fotografía de Edwin Harvey 2012

La ciudad de Corrientes se localiza en el extremo noroccidental de la provincia de Corrientes a los $27^{\circ} 28^{\prime} \mathrm{S}$ y $58^{\circ} 50^{\prime} \mathrm{W}$, sobre la margen izquierda del río Paraná (Figura 2).

El área urbanizada se extiende sobre el extremo occidental de la Lomada Norte del Riachuelo. Dicha lomada se encuentra interpuesta entre el arroyo Riachuelo y el río Paraná, extendiéndose $80 \mathrm{~km}$ desde la ciudad de Corrientes hacia el Este.

Figura 2. Provincias pertenecientes a la Región Norte Grande Argentino y ubicación de la ciudad de Corrientes

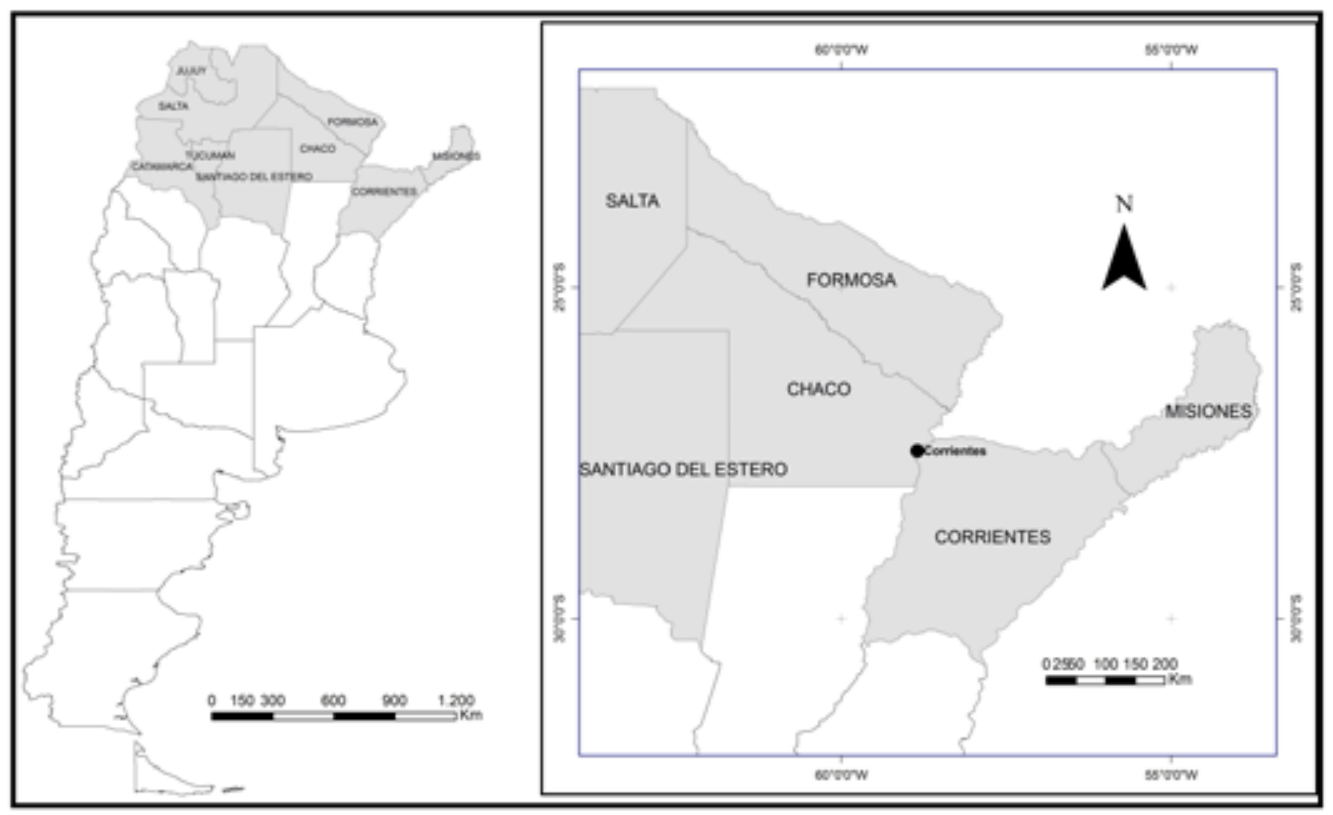

Fuente: Elaboración propia en base a Mignone (2010) 
Contreras y Fantin. El riesgo de la población a inundaciones por lluvias como consecuencia de la dinámica...

Es importante destacar que la población de la ciudad de Corrientes, a lo largo de su historia ha quedado expuesta ante determinados riesgos o ha tenido que sobrellevar ciertas eventualidades desfavorables en la ocupación de nuevos espacios.

Entre 1760 y 1814, según Maeder y Gutiérrez (2003), la ciudad duplicó su población. De 2.514 habitantes estimados en 1769 , pasó a 4.771 en 1814 y a 5.308 en 1820 (Tabla 1). Este crecimiento se manifestó también en la expansión de la planta urbana.

Tabla 1. Población de la ciudad de Corrientes

\begin{tabular}{cccc}
\hline Año & Población & Año & Población \\
\hline 1769 & 1.769 & 1960 & 105.915 \\
\hline 1814 & 4.771 & 1970 & 137.823 \\
\hline 1820 & 5.308 & 1980 & 187.757 \\
\hline 1869 & 1.448 & 1991 & 268.080 \\
\hline 1895 & 21.588 & 2001 & 328.868 \\
\hline 1914 & 39.031 & 2010 & 358.223 \\
\hline 1947 & 71.856 & 2011 & 389.430 \\
\hline
\end{tabular}

Fuente: Maeder y Gutiérrez (2003) y Ramirez et al (2013)

Hacia 1802, al casco urbano se le adjudicaban doce cuadras en dirección este oeste y diez en sentido norte - sur. Dichas cuadras no estaban edificadas en su totalidad, ya que había en ellas extensos baldíos y huertas que tornaban irregular su trazado. Los arroyos Salamanca e Isiró al sudoeste y el Poncho Verde y el Manantiales al este, limitaban la expansión de la ciudad. Ceñida por el río Paraná en el norte, su crecimiento se diluía hacia el sur, donde se hallaba, todavía aislada, la capilla de la Cruz, actualmente en el corazón del centro urbano (Figura 3).

Si bien estos arroyos constituían los límites naturales de la ciudad, el escurrimiento en las calles mal niveladas representaba un importante problema a resolver ya que las aguas abrían profundas zanjas que debían rellenarse periódicamente. En ocasión de las fiestas religiosas, la práctica habitual consistía en colocar vigas atravesadas para impedir que las aguas se llevaran la arena de las calles (Maeder y Gutiérrez, 2003).

Según relatan Gutiérrez y Sánchez Negrete (1988), como las calles y senderos de la ciudad no eran mantenidos de manera sistémica padecían los estragos del trajín de las cabalgaduras y del ganado, así como de las lluvias torrenciales o las inundaciones que los convertían en torrentes y los inhabilitaban con profundos zanjones. Dichos autores también mencionaron que debido a que la ciudad no poseía un adecuado sistema de drenaje y acequias, en tiempos de lluvia se formaban verdaderas "avenidas" de agua que obligaron a levantar las galerías de las casas y a instalar cimientos de piedra más altos para evitar el desmoronamiento de las casas y rancheríos construidos con estanteo o adobe.

Entre 1826 y 1854 se rectificaron las calles y se generó la nomenclatura urbana. La zanja de desagüe que corría al sur, entre las nacientes de los arroyos Limita y 
Manantiales, constituía el límite de la ciudad. Las epidemias de cólera (1868) y de fiebre amarilla (1871) obligaron a clausurar los antiguos cementerios y a habilitar otro al sur de la zanja de desagüe (1873), al que se llegaba en 1875 por un camino precario (Figura 3). Entre 1929 y 1944, se construyó la avenida costanera y, más tarde, el entubamiento de los arroyos Salamanca $(1932$ - 1938) y Poncho Verde (1957 - 1959), la pavimentación de las avenidas 3 de Abril (1949 - 1952) y Maipú (1958 - 1960), permitieron una rápida urbanización, mientras que extensos espacios públicos como el hipódromo y el campo de aviación del Aero Club, se convirtieron en barreras que frenaban la nueva expansión que experimentaba la ciudad (Maeder y Gutiérrez, 2003).

Figura 3. Plano de la Ciudad de Corrientes en 1878

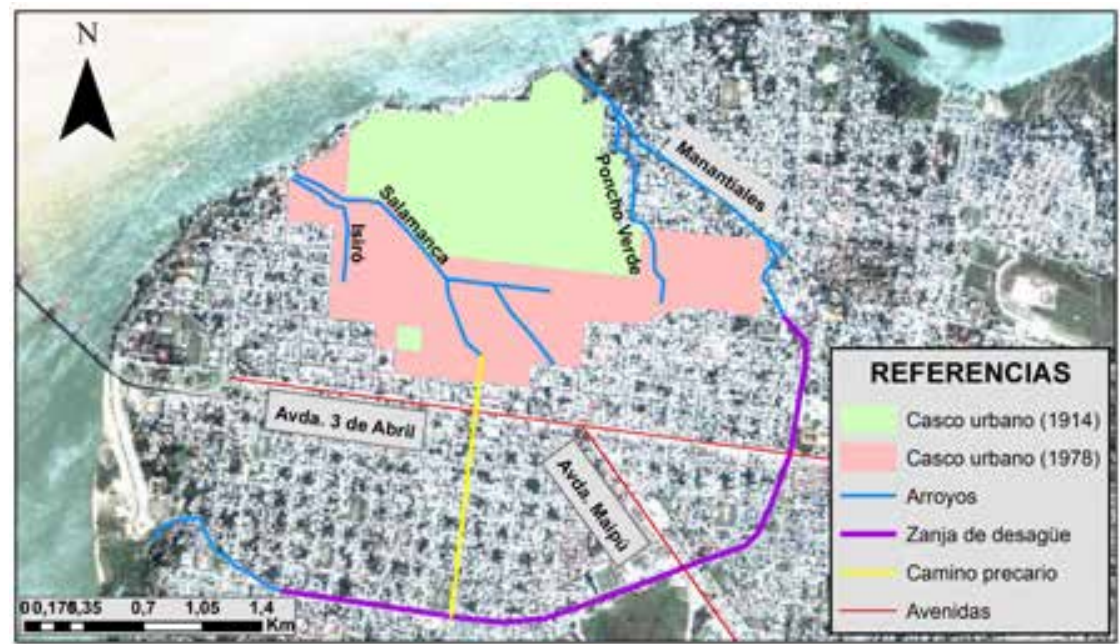

Fuente: Elaboración propia en base a Maeder y Gutiérrez (2003).

\section{Desde 1950 hasta la actualidad}

Como se ha mencionado, la ciudad de Corrientes ha eludido las distintas vicisitudes que se fueron presentando en la ocupación de nuevos espacios. En primer lugar, se esquivaron los distintos arroyos que se encontraban dentro del casco urbano, los cuales posteriormente fueron entubados en vistas de una densificación de la ciudad. Lamentablemente, si bien hoy en día se han realizado obras que mejoran el sistema de desagüe pluvial, los valles de estos arroyos continúan activos y, sumado al encauzamiento producido por las calles y a la saturación de dicho sistema, entre otros; dentro del casco céntrico se forman verdaderos torrentes que traen aparejados importantes problemas a la ciudad en períodos de intensas lluvias.

Por otra parte, fuera del casco histórico de la ciudad, los paisajes se diversifican y aumentan las áreas anegables. La compilación de fotografías aéreas del año 1950, muestra la situación de la ciudad y sus áreas aledañas (Figura 4). Por aquellos años, la ciudad presentaba sus límites donde comienza la superficie anegadiza de la planicie 
Contreras y Fantin. El riesgo de la población a inundaciones por lluvias como consecuencia de la dinámica...

embutida que bordea a la lomada arenosa (Figura 4, delimitada con color amarillo). Por otra parte, se observa cómo la Lomada Norte se encuentra ocupada casi en su totalidad por campos destinados a actividades agropecuarias, que contribuían al abastecimiento de la ciudad, así como un importante número de lagunas, dos de ellas de gran porte.

Al superponer las imágenes satelitales con la compilación fotográfica (georreferenciada mediante Global Mapper 14), se observa que en la actualidad la ciudad ha avanzado ininterrumpidamente ocupando toda la superficie de la Lomada Norte hasta la ruta nacional $\mathrm{N}^{\circ} 12$. En 1950, la ciudad de Corrientes abarcaba una superficie aproximada de $17 \mathrm{Km}^{2}$ y desde entonces, hasta el año 2012, el crecimiento fue de $33 \mathrm{Km}^{2}$, es decir que ahora son $50 \mathrm{Km}^{2}$. En otras palabras, el crecimiento espacial en estos últimos 62 años duplicó el casco urbano al existente en el año 1950.

Figura 4. Fotografía aérea de la ciudad de Corrientes en el año 1950

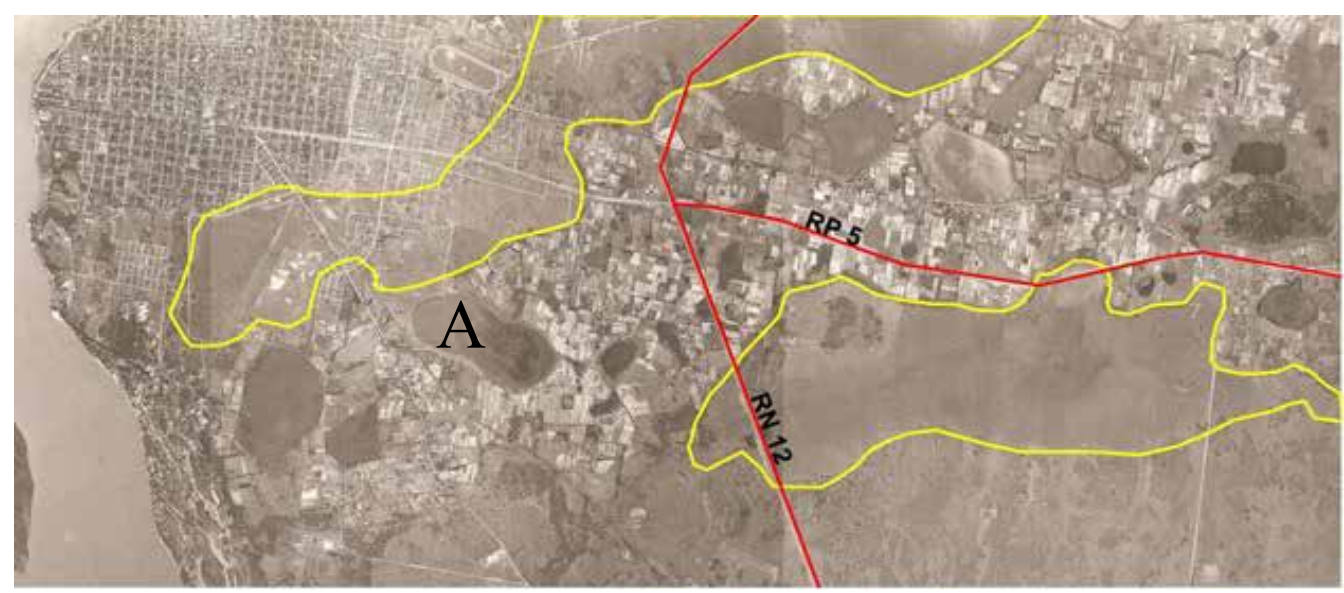

Fuente: Instituto de Limnología Nacional.

Como consecuencia de ello, se han modificado el paisaje y el uso del suelo. En primer lugar, la expansión urbana ha contribuido a la desaparición de 33 lagunas de la Lomada Norte equivalentes a aproximadamente $3,4 \mathrm{Km}^{2}(10 \%$ de los espacios ocupados por el crecimiento espacial). Sobre una de las citadas lagunas de gran tamaño, se ha edificado el barrio llamad "Laguna Seca", el que presenta en la actualidad grandes problemas de anegamiento (Figura $4 \mathrm{~A}$ ).

En síntesis, la ciudad de Corrientes muestra dos situaciones opuestas luego de importantes precipitaciones. Por un lado, la formación de torrentes en los valles de los antiguos arroyos dentro del casco histórico de la ciudad y, por el otro, el estancamiento de las aguas en antiguos lugares ocupados por bañados y lagunas en los nuevos espacios de ocupación. En ambos casos, la población desprevenida queda expuesta a pérdidas materiales (principalmente de vehículos) sin importar su condicional social. No obstante, son los nuevos espacios de ocupación, sin antecedentes previos, donde las pérdidas materiales pueden estar asociadas a las viviendas como se muestra en la figura 5. 
Figura 5. Fotografía de una familia afectada por las lluvias del mes de abril de 2014

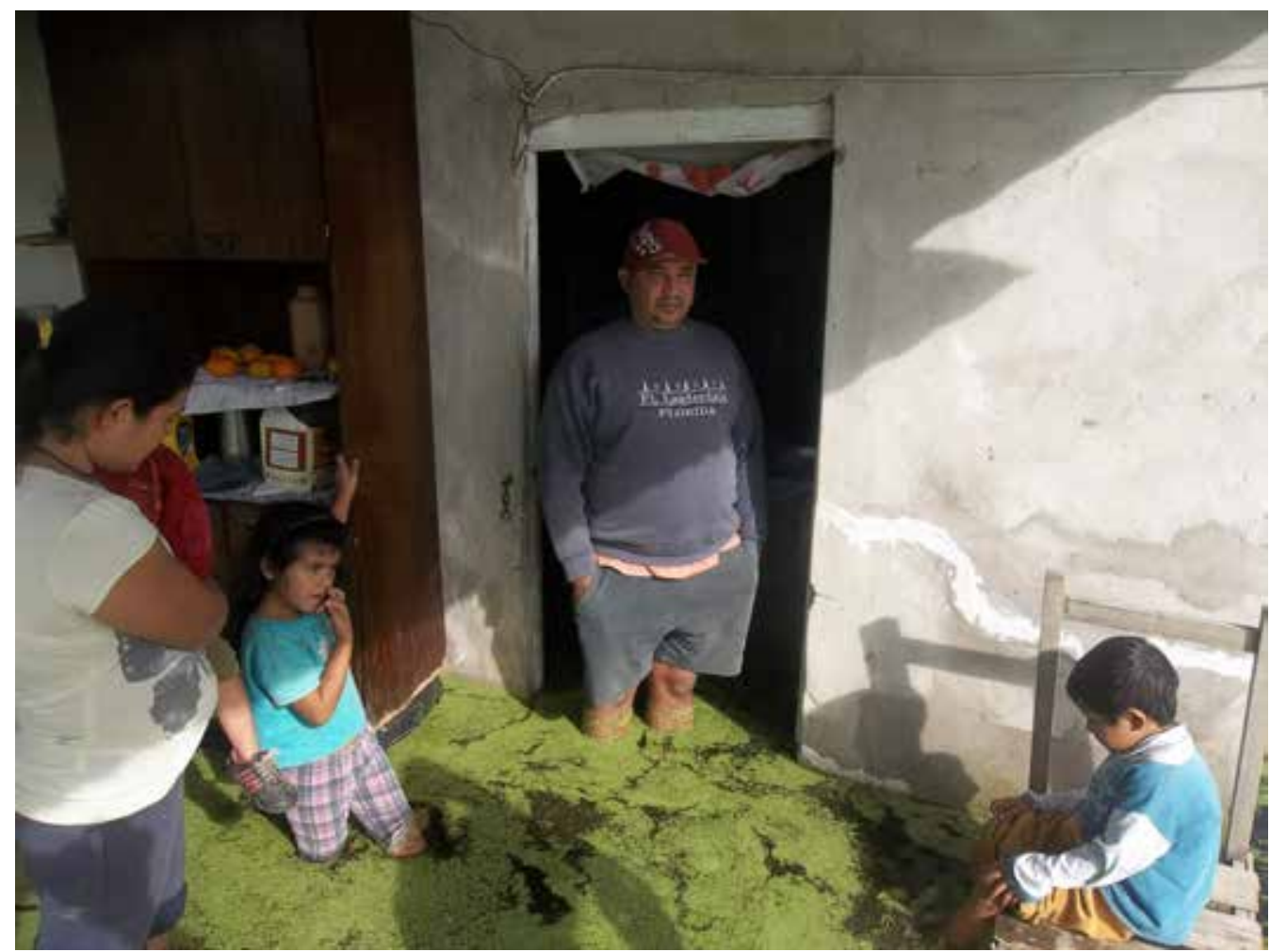

Fuente: Base de datos personal. Mayo de 2014.

Estos últimos son significativos y constituyen un importante antecedente a tomar en cuenta a la hora de planificar la ocupación de nuevos espacios de extensión.

\section{Ocupación de nuevos espacios urbanos}

Para Clichvsky (2006: 35) "Los nuevos procesos económicos, especialmente a partir de los años Noventa del siglo pasado, han traído como consecuencia diferentes formas de inversión en la ciudad. Ha cambiado el submercado residencial. El paralelo a la polarización mayor de la sociedad, este submercado ha respondido con nuevas "mercaderías": viviendas lujosas, para los sectores de más altos ingresos, clubes de campo, barrios cerrados, hasta ciudades privadas, que significan transformaciones territoriales ambientales (modificación hasta de topografías, por grandes movimientos de tierra, por ejemplo, que traen como consecuencia nuevos problemas urbanoambientales: taponamiento de bañados, cambios de escorrentías y, por lo tanto, mayores posibilidades de anegabilidad del territorio que en otros periodos históricos".

En la actualidad, el crecimiento de la ciudad ocurre con una dirección NE y E, a lo largo de la ruta nacional 12 con dirección NE (RN 12) y la ruta provincial 5 (RP 
Contreras y Fantin. El riesgo de la población a inundaciones por lluvias como consecuencia de la dinámica...

5) con dirección E (Figura6). Esta última ruta fue trazada sobre la lomada arenosa y presenta una topografía más elevada. Por este motivo, el principal crecimiento espacial de la ciudad se realiza a lo largo de la RP 5, hasta llegar a la localidad Laguna Brava, que forma parte del área conurbada.

Figura 6. Expansión urbana de la ciudad de Corrientes con dirección NE y E

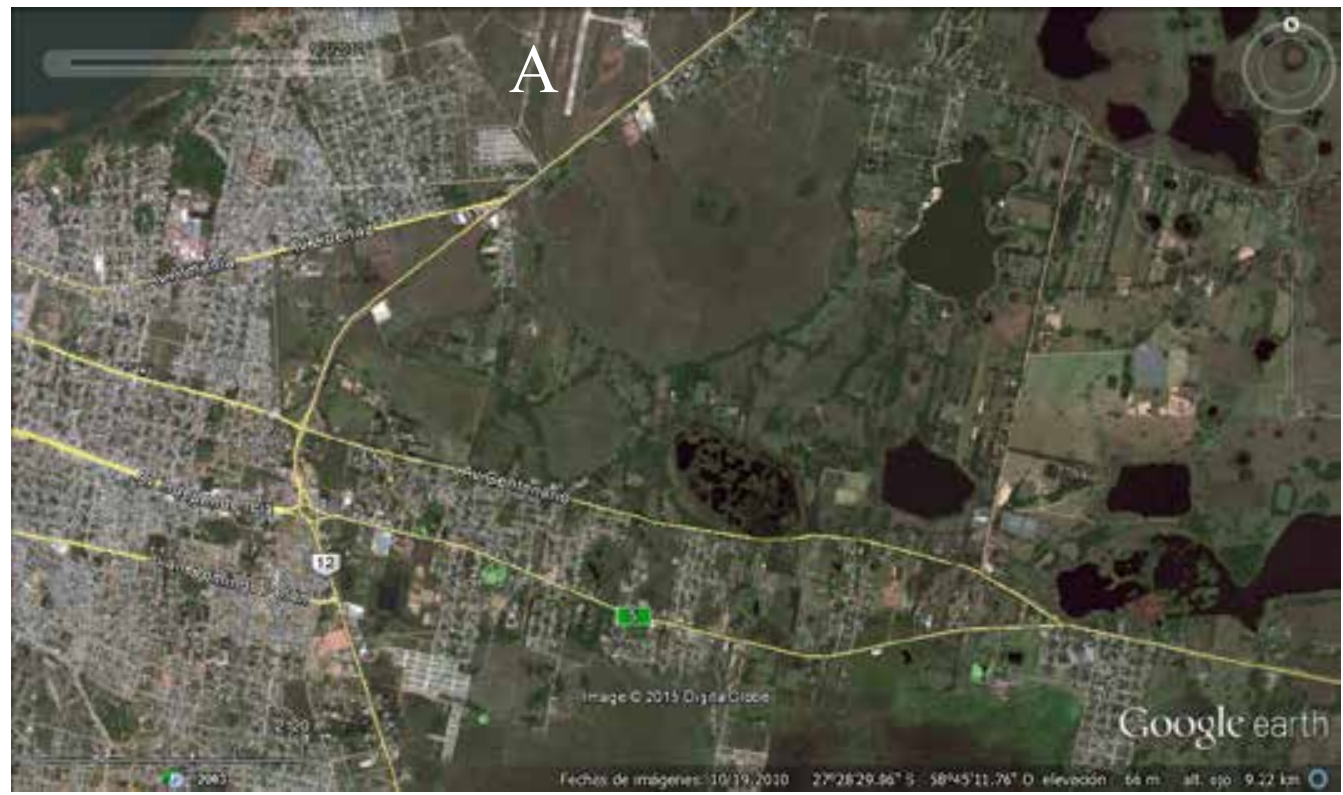

Fuente: Imagen de Google Earth.

La figura 7 muestra cómo, en la actualidad, el límite del crecimiento de la ciudad evita los espacios desfavorables, ya que las manzanas, calles y casas que se observan se ubican sobre el límite sur de la Lomada Norte, dando comienzo a un paisaje de esteros. En otras palabras, la tendencia actual, dado a que todavía existen espacios sobre la lomada arenosa, es la de crecer siguiendo su ubicación, motivo por el cual el barrio Laguna Brava en poco tiempo se anexaría al casco urbano de la ciudad de Corrientes.

El aeropuerto actúa como límite antropogénico a la expansión de la ciudad sobre el frente con dirección NE (Figura 6, punto A). Por otra parte, hacia el sur entre la RN 12 y la RP 5, gran parte del área está ocupada por bañados, siendo éste el principal motivo por el cual dicha zona se encuentra despoblada. A pesar de ello, se observa se están trazando calles sobre estos espacios, con una fuerte demanda ocupacional, ya que los costos de los mismos son marcadamente inferiores en relación a los ofrecidos sobre la lomada. 
Figura 7. Límite natural de la expansión con dirección E de la ciudad de Corrientes

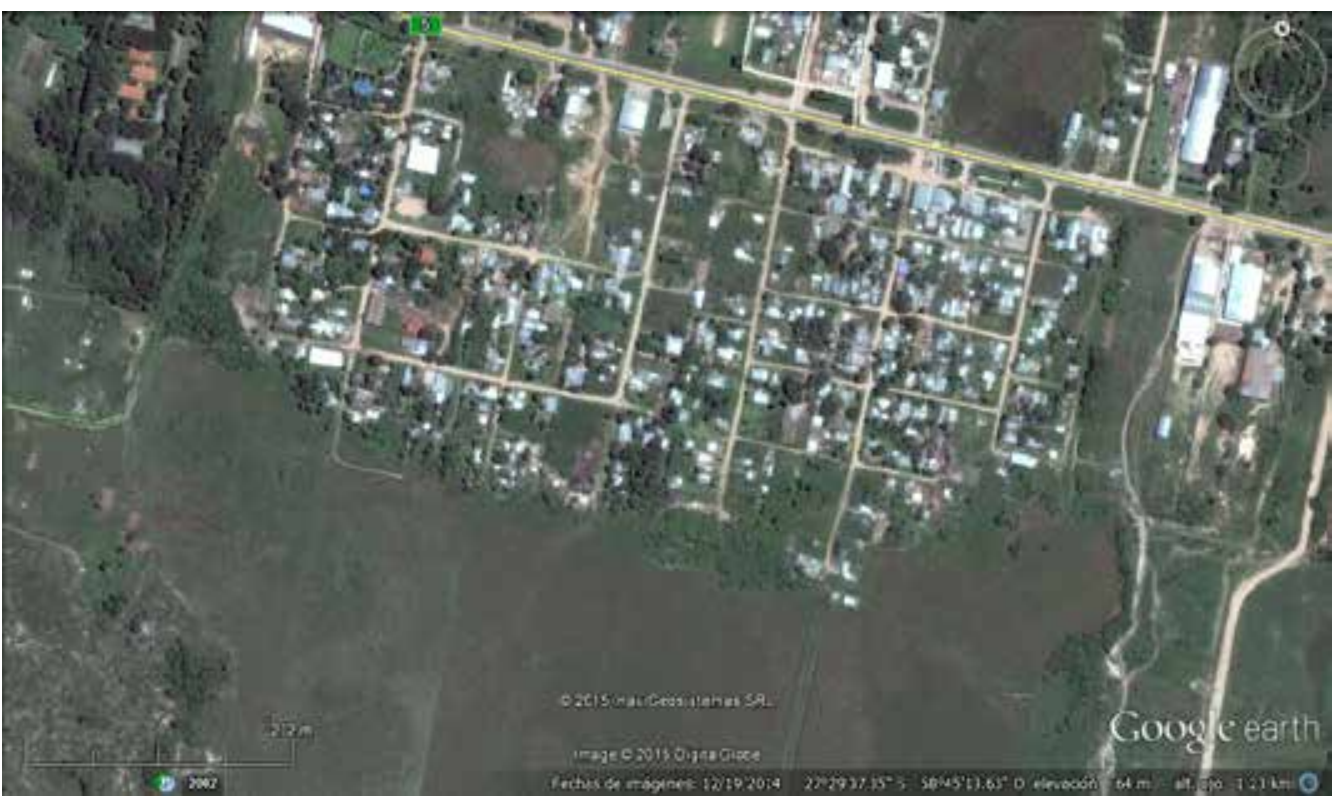

Fuente: Imagen de Google Earth.

Debido a que la ocupación de dichos terrenos no ha sido acompañada por la realización de obras de desagüe pluvial, las viviendas que allí se construyen presentan serios inconvenientes de anegamiento en caso de precipitaciones abundantes, motivo por el cual se deben construir sus cimientos con una altura superior a 1,2 m (figura 8).No obstante, éste no es el único inconveniente que se presenta, ya que las intensas lluvias también ocasionan que las calles de acceso a dichos terrenos, delimitadas sobre suelos arcillosos, se tornen intransitables dejando a las viviendas aisladas por los menos una semana.

Figura 8. Construcción de una vivienda sobre un paisaje de bañados secos



Fuente: Base de datos personal. Junio de 2013. 
Contreras y Fantin. El riesgo de la población a inundaciones por lluvias como consecuencia de la dinámica...

En lo que respecta a la expansión de la ciudad de Corrientes hacia el sur, la situación es similar a la ya mencionada. Se destacan grandes espacios "vacíos", los que corresponden principalmente a áreas de bañados que se alternan con algunos sitios relativamente más elevados y en donde aparecen espacios ocupados por sabanas arbóreas o con bosquecillos poco densos de Prosopis affinis y espartillares de Elyonurus muticus (Carnevalli, 1994).

La ciudad avanza en los distintos frentes por medio de barrios planificados para habitantes de clase media, los que fueron erigidos en espacios que presentan condiciones favorable para el drenaje, generando óptimos espacios para el asentamiento, aunque otros, como el barrio planificado Laguna Seca, fueron trazados y construidos sobre terrenos previamente ocupados por lagunas. Por esta razón, se detectan fuertes contrastes. Por un lado, la presencia de asentamientos espontáneos en las grandes áreas pertenecientes a bañados, donde la precariedad de las viviendas sumada a las condiciones desfavorables del terreno, como ser el déficit de escurrimiento, dejan expuestas a la población a condiciones ambientales que favorecen la aparición de ciertas enfermedades (por ejemplo el dengue). La falta de servicios mínimos como ser agua, luz, salud y transporte potencian dicha vulnerabilidad social (Figura 9). En el otro extremo, se encuentran los espacios que ofrece la Lomada Norte, que son de elevado costo y que se ofrecen como futuros barrios privados o clubes de campo.

Figura 9. Asentamiento espontáneo sobre bañados luego de precipitaciones

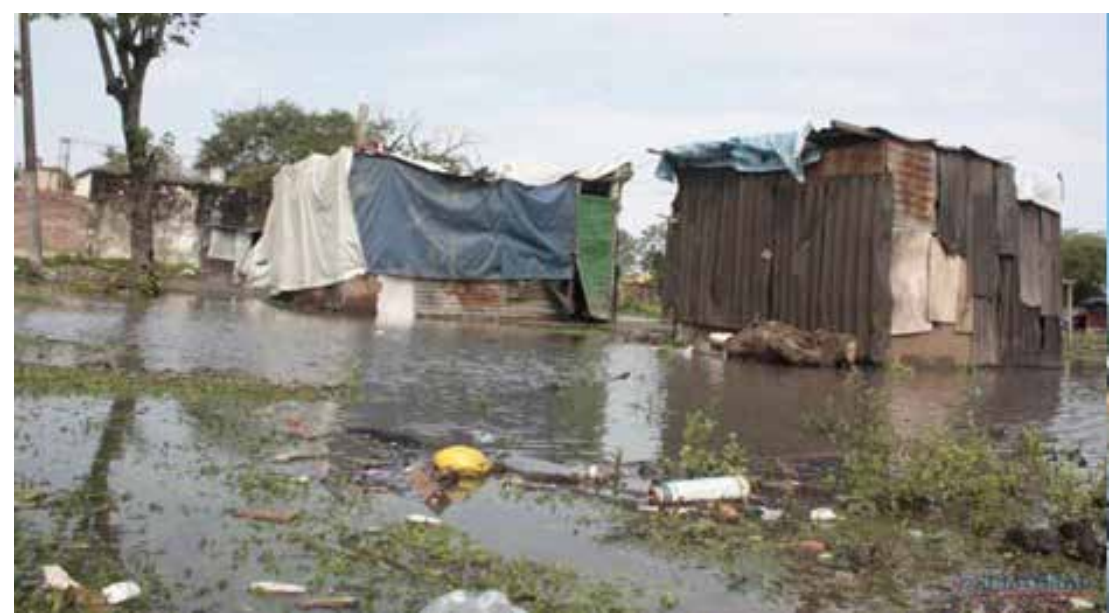

Fuente: http://www.ellitoral.com.ar/es/articulo/224584/Galvan-okupas-con-terrenos-anegados--y-carpasinundadas-por-la-tormenta

No obstante, la expansión urbana sobre la lomada no ofrece garantías que aseguren un lugar propicio para el asentamiento. En ella existen grandes lagunas, algunas utilizadas como espacio de recreación o en otros casos, como atractivo paisajístico de los barrios privados; pero también se encuentra un gran número de pequeñas cubetas de escasa profundidad y con limitada capacidad de retención de agua, motivo por el 
cual desaparecen en períodos de sequía dejando espacios libres de vegetación. Es precisamente sobre estos lugares donde la población se instala, quedando expuesta a situaciones adversas luego de importantes precipitaciones (Figura 10). En dicha figura se muestran las imágenes satelitales de un mismo terreno capturadas en años diferentes. En la imagen del año 2003 se observan dos cubetas someras y otras dos más profundas además de un espacio poco poblado. Sin embargo, para el año 2005 ya se observa la construcción de una casa sobre la laguna (Fig. 10 laguna B) ocupando el 25\% de la cubeta, además de elevar el terreno, modificando la morfología de la cubeta; situación que se repite en la laguna A desde el año 2012.

Es evidente que la ocupación de estos espacios anegadizos se manifiesta en períodos secos, ya sean bañados o lagunas; estos humedales se secan por completo por falta de lluvias, dejando grandes extensiones de tierra descubierta que, como se destacó anteriormente, son aprovechadas por la especulación inmobiliaria. En este sentido, Clichvsky (2006: 35) señala que "la inundación afectará al mercado de tierra en su totalidad (incluso fuera del centro urbano) en cada uno de sus submercados en mayor o menor medida dependiendo de su dinámica y organización específica. Puede producirse una paralización total de todos o algunos submercados o sólo una baja en los precios más o menos importante, en un período acotado de tiempo"; es por ello que se deben agudizar los controles del mercado inmobiliarios en ciudades como Corrientes, donde los paisajes aledaños son anegadizos y donde se registran prolongados períodos secos.

Figura 10. Modificación del paisaje de lagunas

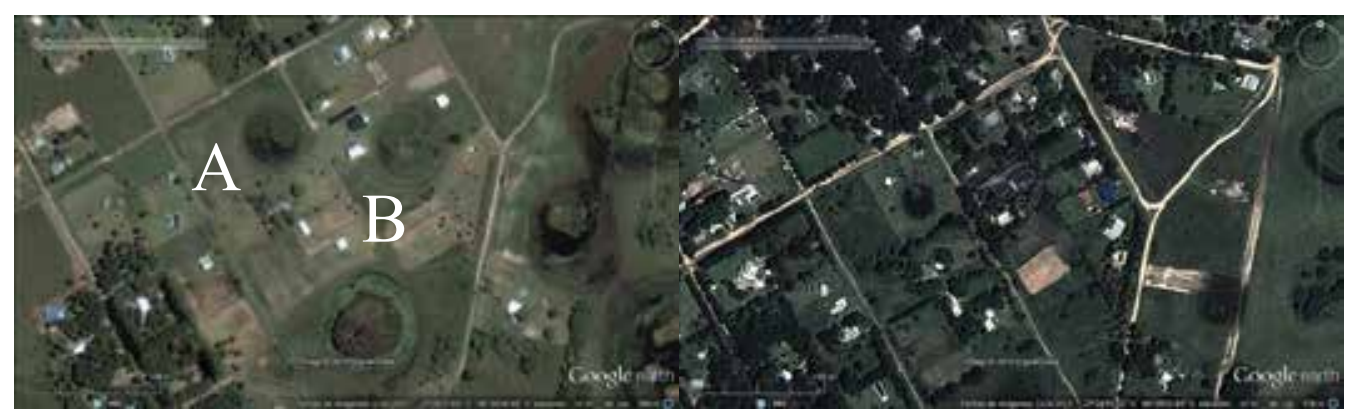

Fuente: Imagen de Google Earth.

Esta última figura evidencia la urgencia de generar políticas de crecimiento urbano intentando reducir al mínimo los posibles impactos naturales y los riesgos a los que la población de la ciudad de Corrientes se encuentra expuesta. Sin dudas las mejores condiciones naturales para el crecimiento de la ciudad se presentan, en primer término, hacia el este, sobre la Lomada Norte, preservando sus lagunas, ya que no solo pueden constituir un elemento de realce paisajístico, sino que a su vez actúan como reservorio de agua, que de tener en cuenta su ubicación pueden reducir al mínimo los riesgos por anegamiento. 
Contreras y Fantin. El riesgo de la población a inundaciones por lluvias como consecuencia de la dinámica...

\section{Conclusión}

Es evidente que la expansión urbana genera un impacto en la naturaleza por lo que resulta de fundamental importancia contar con políticas de ordenamiento territorial que actúen como reguladoras y controladoras de los frentes de avance de una ciudad, más aún cuando las condiciones naturales pueden dejar expuestas a la población a determinados riesgos.

La ciudad de Corrientes no presenta lugares propicios para su expansión en todas las direcciones, esto conlleva a que aquellos sitios más favorables sean muy demandados y por consiguiente aumente el valor de la tierra asociado a la especulación inmobiliaria. Como consecuencia de ello, se ocupan espacios que naturalmente son anegables, aumentando consigo la exposición de la población luego de lluvias intensas frente a riesgos de pérdidas materiales, de difícil o imposible acceso y/o movilidad y que en algunos casos, puede determinar la carencia de servicios básicos. En este sentido, se observan tres situaciones de expansión: A través de barrios privados ubicados sobre la Lomada Norte que presenta las mejores condiciones para ello; barrios planificados ubicados en espacios que se encuentran en áreas con condiciones intermedias y por último los asentamientos espontáneos, que se ubican sobre áreas inundables que rodean a la ciudad.

Las superficies con esteros y bañados son las principales limitantes en la expansión urbana de la ciudad de Corrientes, aunque son superadas bajo la presión inmobiliaria ejercida por la demanda en la ocupación de nuevos espacios.

Históricamente la ciudad ha mostrado distintos problemas luego de producirse intensas precipitaciones, no obstante se pueden diferenciar dos situaciones principales. Por una parte, el casco histórico de la ciudad presenta escurrimientos torrenticos en las calles trazadas sobre antiguos arroyos. Por la otra, fuera del casco histórico, la ocupación de espacios anegadizos, ya sea sobre las planicies embutidas o sobre los que pertenecían a las lagunas de la Lomada Norte, genera importantes volúmenes de agua estancada en distintos barrios y vías de comunicación de la ciudad. De todas maneras, ambas situaciones están relacionadas con el riesgo de pérdidas materiales, principalmente de vehículos, mientras que en la ocupación de nuevos espacios, sin antecedentes, las pérdidas materiales se encuentran relacionadas a daños en las viviendas, sin importar la condición social de la población expuesta.

Por los motivos antes expuestos, resultan de interés los antecedentes históricos de la ciudad y los estudios multidisciplinarios con el objeto de lograr un ordenamiento territorial y de evaluar los riesgos a los que está sometida la población, los impactos de la misma e incluso los tiempos de recuperación del espacio geográfico expuesto al impacto, de modo de tener en cuenta todas las variables posibles a ser consideradas y así reducir al mínimo tanto la vulnerabilidad de la población como las alteraciones que generen las acciones antropogénicas al paisaje. 


\section{Referencias bibliográficas}

Barrenechea, J.; Gentile, E.; González, S. y Natenzon, C. 2003. "Una propuesta metodológica para el estudio de la vulnerabilidad social en el marco de la teoría social del riesgo". En: Lago Martínez, S. Gómez Rojas G. y Mauro, M. (Coord.). En torno de las metodologías: abordajes cualitativos y cuantitativos. Buenos Aires, Proa XXI, pp. 179-196.

Blaikie, P.; Cannon, T.; Davis, I. y Wisne, B. 1998. Vulnerabilidad. El entorno social, político y económico de los desastres. Bogotá, la RED/ITDG.

Carnevali, R. 1994. Fitogeografia de la provincia de Corrientes. Asunción, Paraguay, Editorial Litocolor, p. 123.

Chardon, A. 1998. "Crecimiento urbano y riesgos "naturales": Evaluación final de la vulnerabilidad global en Manizales, Andes de Colombia". Desastres y Sociedad. Red de Estudios Sociales. En: Prevención de Desastres en América. No 9, pp. 5-35.

Clichvsky, N. 2006. "Estado, mercado de tierra urbana e inundaciones en ciudades Argentinas". Cuadernos de Geografía, Universidad Nacional de Colombia. $\mathrm{N}^{\mathrm{o}}$ 15 , pp. $31-52$.

Giddnes, A. 1990. Consecuencias de la modernidad. Madrid, Alianza Editorial.

Gutiérrez, R. y Sánchez Negrette, A.. 1988. Evolución urbana y arquitectónica de Corrientes. Buenos Aires, Instituto Argentino de investigaciones en Historia de la Arquitectura.. Tomo I.

Maeder, E. y Gutiérrez, R. 2003. Atlas del desarrollo Urbano del Nordeste Argentino. Resistencia, Instituto de Investigaciones Geohistóricas-CONICET, Universidad Nacional del Nordeste, p. 180.

Meichtry, N. y Mignone, A. 2003. "Movilidad territorial. Una contribución a partir de la población en asentamientos periféricos espontáneos en Resistencia, Chaco, en la década de 1990". En: VI Jornadas Argentinas de Estudios de la Población. Buenos Aires, Asociación de Estudios de Población de la Argentina, pp. 324-339.

Mignone, A. 2008. "Las condiciones de pobreza en las capitales del nordeste argentino a principios del siglo XXI. Su análisis a través del Índice de Privación Material de los Hogares (IPMH)”. En XXVII Encuentro de Geohistoria Regional. Resistencia, Instituto de Investigaciones Geohistóricas CONICET. Agosto $28-30$.

Mignone, A. 2010. "La segregación por pobreza en las capitales provinciales del Norte Grande Argentino. Un análisis cuantitativo a partir del IPMH”. Breves Contribuciones del I.E.G. Instituto de Estudios Geográficos de la Facultad de Filosofía y Letras de la Universidad Nacional de Tucumán. $\mathrm{N}^{\mathrm{o}}$ 21, p. 40-59.

Neiff, J. Humedales de la Argentina: sinopsis, problemas y perspectivas futuras. URL: http://www.neiff.com.ar/documentacion/03.\%20Capitulos $\% 20 \mathrm{de} \% 201 \mathrm{ibro} /$ ARCHIVO\%2034.\%20Neiff,\%202001b.pdf. Consultado el 10 de abril de 2015

Olcina Cantos, J. 2004. "Riesgo de inundaciones y ordenación del territorio en la escala local. El papel del planeamiento urbano municipal”. Boletín de la A.G.E. No 37 , p. 49-84. 
Ramírez, M.; Monzón, N.; Claret, R., Contreras, F. y Belingheri, B. 2013. Corrientes en cifras. Recopilación y compilación estadística para la interpretación de la situación actual. Resistencia, Chaco, Ed. Contexto, p. 432.

Ringuelet, R. 1962. Ecología Acuática Continental., Bs. As. Ed. Eudeba.

Terradas, J.; Franquesa, T.; Parés, M. y Chaparro, L. 2011. "Ecología urbana". URL: http://www.conciencia.herobo.com/wordpress/wp-content/uploads/2012/03/ Ecolog\%C3\%ADa-urbana.pdf Consultado el 10 de abril de 2015. 